\title{
CARACTERÍSTICAS DEL TAMIZAJE PARA CÁNCER CÉRVICO-UTERINO EN 08 ESTABLECIMIENTOS DE SALUD, LIMA METROPOLITANA 2017
}

\author{
CHARACTERISTICS OF THE CERVICO UTERINE CANCER SCREENING AT 08 HEALTH INSTITUTIONS, \\ METROPOLITAN LIMA 2017
}

Cvetkovic-Vega, A. ${ }^{1, a, b}$, Carlos León-Nakamura ${ }^{1, a, b}$, Alejandro Yábar-Berrocal 1,a,b

\begin{abstract}
RESUMEN
Introduccion: El uso de Citología Convencional de Papanicoloau (CC) frente a Base Líquida (CBL), el profesional que realiza la extracción, primera lectura y diagnóstico de la muestra; el uso de la clasificación Bethesda, la detección de ADN del VPH y el seguimiento de hallazgos ASCUS son eslabones en el tamizaje del Cáncer del Cuello Uterino (CCU) poco estudiadas en nuestro medio. Objetivo: Determinar las características del tamizaje para cáncer CCU en 08 establecimientos de salud (EESS) de Lima Metropolitana sedes del Internado Médico de la FAMURP en el 2017.Métodos: Descriptivo, transversal. Muestreo por conveniencia. Se entrevistó a los Jefes de los Servicios de Patología. Resultados: Participaron 8 EESS de Nivel III del MINSA, EsSalud y FFAA. Todos realizaron la CC, ninguno CBL. Las muestras son extraídas por el Ginecólogo o la Obstetriz, y en 1 caso por enfermería, la primera lectura es realizada por el Tecnólogo Médico y en un caso por el Anátomo Patólogo; el diagnóstico, por el Anátomo Patólogo. Se usa el Sistema de Bethesda para el Reporte Final. No se usan pruebas de detección para ADN del VPH. En 8, se hace seguimiento para hallazgos ASCUS. Conclusión: La CBL, la lectura automatizada, y la identificación del ADN de VPH no están implementados. Se recomienda realizar estudios costo-efectivo para proponer su implementación futura, y realizar estudios respecto de las fases del proceso del Tamizaje.
\end{abstract}

Palabras clave: Prueba de papanicolaou; Citología; Cáncer cérvico uterino; PVH; Tamizaje. (fuente: DeCS BIREME)

\begin{abstract}
Introduction: Use of Papanicolaou's test versus Liquid Based Cytology; professional that executes the sample's extraction, first lecture and diagnosys; use of Bethesda clasification; VPH's DNA detection, and the follow up of ASCUS results, are important topics of the Cervical Cancer screening which are scarcely studied at Peru. Objective: To determinate characteristics from the screening of Cervical Cancer at 08 Health institutions (HI) from Metropolitan-Lima in 2017 which were Intership Medical Centers for FAMURP students. Methods: Observational, descriptive transversal study with convenience type sampling. Pathology's Chiefs from HI were interviewed. Results: 8 Third level HI from MINSA, ESsalud and FFAA were included. All HI use Papanicolaou Test, no LBC were used. Samples were taken by the Gynaecologist or the Obstetrician and in one case by the nurse; first lecture, by the Medical Tecnologyst and in one case by the anatomic pathology specialist; diagnosys, by the anatomic pathology specialist. The Bethesda System was used at the Final Report. No HPV- DNA detection test was used. $8 \mathrm{HI}$ made ASCUS finding follow up. Conclusion: LBC, automatic lecture for screening and HPV-DNA detection are not implemented. It is recommended to desing cost-effective studies for their future implementation, and desing studies at the Cervicuterine cancer Screening stages.
\end{abstract}

Key words: Papanicolaou Test; Liquid-based Cytology; Cervicuterine Cancer; HPV; Screening. (source: MeSH NLM)

\footnotetext{
${ }^{1}$ Hospital Nacional Hipólito Unanue, Lima-Perú.

${ }^{2}$ Instituto de Investigación en Ciencias Biomédicas, Universidad Ricardo Palma, Lima - Perú.

a Médico Cirujano.

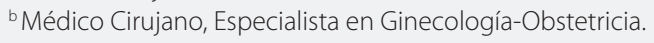

‘Médico Cirujano, Especialista en Anatomía Patológica.
}

Citar como: Cvetkovic-Vega, A., Carlos León-Nakamura, Alejandro Yábar-Berrocal. Características del tamizaje para cáncer cérvico-uterino en 08 establecimientos de salud, lima metropolitana 2017. [Artículo Original].2019;19(1):16-23. (Enero 2019). DOI 10.25176/RFMH.v19.n1.1788 


\section{INTRODUCCIÓN}

El cáncer cérvico-uterino (CCU) es una patología causada principalmente por los subtipos oncogénicos 16 y 18 del Papiloma Virus Humano (PVH)', siendo el cuarto cáncer más frecuente a nivel mundial ${ }^{2}$. La incidencia del CCU en los países varía por cada mil, en Bolivia (36.4), Perú (34.5) en contraste con países desarrollados como Canadá (6.6) y Estados Unidos (EE.UU) (5.7) notándose un claro patrón entre la incidencia y el grado de desarrollo del país ${ }^{3}$. En el Perú es la primera causa de muerte por Cáncer en general ${ }^{4}$ y en mujeres ${ }^{5}$ ocasionando la pérdida de 378 mil años de vida saludables equivalentes a 900 millones de dólares por año, estableciéndose al CCU como uno de los que más genera años de vida saludables perdidos y pérdidas económicas ${ }^{6}$.

El efecto citopático de la infección por $\mathrm{VPH}^{1}$ se evidencia por la detección oportuna ${ }^{7}$ e idealmente en etapas asintomáticas ${ }^{8}$ de lesiones precancerosas que, según organismos internacionales como la OMS, deben de ser clasificadas y reportadas a través del Sistema de Bethesda ${ }^{3,5}$ actualizado al 2014,9, recayendo esta responsabilidad en el Especialista Médico Citopatólogo ${ }^{10}$. Dicha detección se realiza como parte de un programa de Tamizaje que incluye métodos como la detección del ADN del VPH cuyo hallazgo es definitivo; Citología convencional de Papanicolaou (CC) que a pesar de estar al alcance del primer nivel de atención en el país ${ }^{7}$ tiene limitaciones intrínsecas ${ }^{10}$ que se suman al problema de la cobertura y del sistema de salud per $\mathrm{se}^{11}$; y Citología de base líquida con ventajas establecidas como la reducción en un $70-90 \%$ del índice de muestras no satisfactorias encontradas con la Citología Convencional ${ }^{10}$, pero cuyos costos de implementación aparentemente son su principal limitación.

El estudio de las anormalidades en la citología epitelial que se realiza como parte del proceso de Tamizaje para CCU implica etapas que involucran la toma de muestra, primer lectura y diagnóstico final; el primero es considerado como un proceso fundamental en el que muchos estudios identifican errores en este nivel $^{12}$ a causa de tomadores ocasionales ${ }^{13}$ con repercusiones directas en el aumento del índice de falsos negativos ${ }^{14}$. La primera lectura y diagnóstico final comprende la descripción de células escamosas y las glandulares, haciendo especial énfasis en el primer grupo, correspondiente si fuera el caso a lesiones escamosas intraepiteliales de bajo (LSIL) o de alto (HSIL) grado, existiendo la posibilidad de un grupo de características atípicas de significado indeterminado (ASCUS) y aquellas en las que no pueden excluirse lesiones de alto grado (ASC-H) ${ }^{15}$. Este grupo ASCUS constituye un grupo de interés por su malignidad potencial ${ }^{16}$ y constituyen un grupo no concluyente reportado por el Citopatólogo.

Por todo lo anterior, se evidencia que el tipo de tamizaje, el uso de la clasificación de Bethesda, el profesional relacionado con la toma, primera lectura e interpretación de la muestra, la identificación del ADN del VPH y el seguimiento de hallazgos ASCUS son características importantes del proceso de tamizaje que deben de ser descritas y posteriormente estudiadas a profundidad en nuestra realidad motivo por el cual, y ante la escasa evidencia científica reportada en nuestro país son el objetivo de la descripción en este estudio.

\section{MÉTODOS}

\section{Diseño del estudio}

Estudio observacional, descriptivo transversal.

\section{Población y muestra}

Establecimientos de Salud de Lima Metropolitana que fueron Sede Oficial para el Internado Médico de los alumnos de la Facultad de Medicina de la Universidad Ricardo Palma durante el 2017. El muestreo fue por conveniencia, tomando como unidad de análisis al Servicio de Patología de cada Establecimiento de Salud representado a través de su respectivo Jefe de Departamento o su equivalente. Como criterios de inclusión plantearon (Ver Figura 1): 1) Establecimientos de salud que cuente con Servicio de Anatomía Patológica y de Ginecología; criterios de exclusión: 1) Servicios de Anatomía Patológica que derivan las muestras a otros establecimientos de salud para lectura de láminas; 2 ) Servicio de Anatomía Patológica cuya ficha de recolección de datos esté incompleta; y 3) Servicio que no autorice la realización de la investigación.

\section{Variables}

Mediante revisión de la literatura nacional e internacional y por opinión de expertos en el tema, se plantearon las siguientes variables como representativas en el proceso del Tamizaje para CCU: Tipo de citología (Citología Convencional; Citología de Base Líquida), Profesional que extrae la muestra (Médico Cirujano Especialista, Licenciado en Obstetricia, Licenciada en Enfermería), Profesional que realiza la primera lectura (Anátomo Patólogo, Tecnólogo Médico, Automatizado), Profesional que realiza el diagnóstico (Anátomo Patólogo, Tecnólogo 
Médico, Automatizado), Uso de la Metodología de Bethesda para el reporte final, Detección de ADN de VPH, y Seguimiento de casos con hallazgos ASCUS.

\section{Procedimientos}

El instrumento utilizado fue una ficha de recolección de datos revisada por los asesores temáticos. Se realizaron entrevistas autorizadas con los Jefes de Servicio de Patología de los EESS, Los datos recolectados fueron depurados y ordenados, siendo registrados en una hoja de cálculo de Excel que fue revisada 03 veces para evitar el error. Luego, la base generada fue procesada por un programa estadístico para su análisis.

\section{Ética de la investigación}

Se siguieron los lineamientos internacionales para el adecuado uso de datos y la conservación de la ética en la investigación. El protocolo fue aprobado por el Comité de Ética Institucional e Investigación del Hospital Nacional Docente Madre Niño San Bartolomé. Posteriormente, se contó con la autorización institucional de las 08 Sedes Hospitalarias que aceptaron participar en el estudio, estando de acuerdo en la posterior publicación de los resultados.

\section{Análisis de datos}

Los datos fueron procesados con el paquete estadístico Stata ${ }^{\circledR}$ 11,1 (Stata Corp. Texas, US) para Windows. Las variables cualitativas se analizaron usando frecuencias y porcentajes.

\section{RESULTADOS}

Entre las características generales de los 8 Establecimientos de Salud (EESS) participantes del estudio, se obtuvo que de acuerdo al segmento 5 pertenecían al MINSA, 1 a EsSalud y 2 a las Fuerzas Armadas del Perú. Todos estos establecimientos desarrollan un nivel de Atención Terciario y son de Categoría Nivel III-1, de nivel de Complejidad 7. Adicionalmente, 3 EESS estaban ubicados en el distrito de Jesús María, y los demás en los distritos de Cercado de Lima, El Agustino, San Juan de Miraflores, Pueblo Libre, Comas y Miraflores. (Ver Tabla 1).

Respecto de las características del Tamizaje de Cáncer de Cuello Uterino, se encontró que en todos los EESS, no existiendo diferencia según el segmento, se realiza el Papanicolaou Convencional negando en todos los casos el uso de la Citología de Base Líquida. Sobre los profesionales que extraen la muestra, en 4 EESS dichas muestras son extraídas tanto por Médicos Cirujanos Especialistas en Ginecología y/o por Licenciadas en Obstetricia, siendo el caso que en solo 1 EESS del Segmento MINSA dicha labor está asignada exclusivamente a las Licenciadas de Obstetricia. En un EESS la extracción la realizan tanto el Médico Cirujano Especialista, las Licenciadas en Obstetricias y las Lic. en enfermería. (Figura 1) Sobre el profesional que realiza la primera lectura, en el 100\% Ios EESS las realiza el Tecnólogo Médico, negando en todos los casos y en todos los segmentos que exista un sistema automatizado para dicha lectura. Respecto del profesional que realiza el diagnóstico, dicha labor está en todos los casos a cargo del Anátomo Patólogo. Respecto del Uso del Sistema de Bethesda para el Reporte Final, el $100 \%$ de EESS indicó utilizarlo en su informe. Ningún EESS reportó realizar pruebas para detección de ADN delVPH en las muestras delTamizaje. Respecto del seguimiento de hallazgos ASCUS, dicho proceso se realizó en 7 EESS. (Ver Tabla 2)

Tabla 1. Características generales de los establecimientos de salud participantes.

\begin{tabular}{|c|c|c|}
\hline & $\mathbf{N}^{\circ}$ & $\%$ \\
\hline \multicolumn{3}{|l|}{ Segmento } \\
\hline MINSA & 5 & 62.5 \\
\hline EsSALUD & 1 & 12.5 \\
\hline Fuerzas Armadas del Perú & 2 & 25.0 \\
\hline \multicolumn{3}{|l|}{ Nivel de Atención } \\
\hline Terciaria & 8 & 100 \\
\hline \multicolumn{3}{|l|}{ Categoría de EESS } \\
\hline Nivel III-1 & 8 & 100 \\
\hline \multicolumn{3}{|l|}{ Nivel de Complejidad } \\
\hline VII & 8 & $100 \%$ \\
\hline \multicolumn{3}{|l|}{ Distrito } \\
\hline Cercado de Lima & 1 & 12.5 \\
\hline El Agustino & 1 & 12.5 \\
\hline San Juan de Miraflores & 1 & 12.5 \\
\hline Pueblo Libre & 1 & 12.5 \\
\hline Comas & 1 & 12.5 \\
\hline Jesús María & 2 & 25 \\
\hline Miraflores & 1 & 12.5 \\
\hline
\end{tabular}


Tabla 2. Características del tamizaje de cáncer de cuello uterino en los establecimientos de salud por segmento.

\begin{tabular}{|cccc} 
& \multicolumn{3}{c}{ SEGMENTO (N=8) } \\
& MINSA & ESSALUD & FFAA \\
& $(\mathrm{N}=5)$ & $(\mathrm{N}=1)$ & $(\mathrm{N}=2)$
\end{tabular}

Tipo de citología

Papanicolaou convencional

5

1

2

Citología de Base Líquida

No se realiza en los EESS

Extrae la muestra

Médico Cirujano

Licenciado Obstetricia

Licenciado en enfermería

4

5

0

0

5

No se realiza en los EESS

5

No lo realiza

No se realiza en los EESS

5

1

2

No se realiza en los EESS

5

1

Establecimientos de salud que son Sede Oficial para el internado Médico de los alumnos de la FAMURP en Lima Metropolitana en el 2017
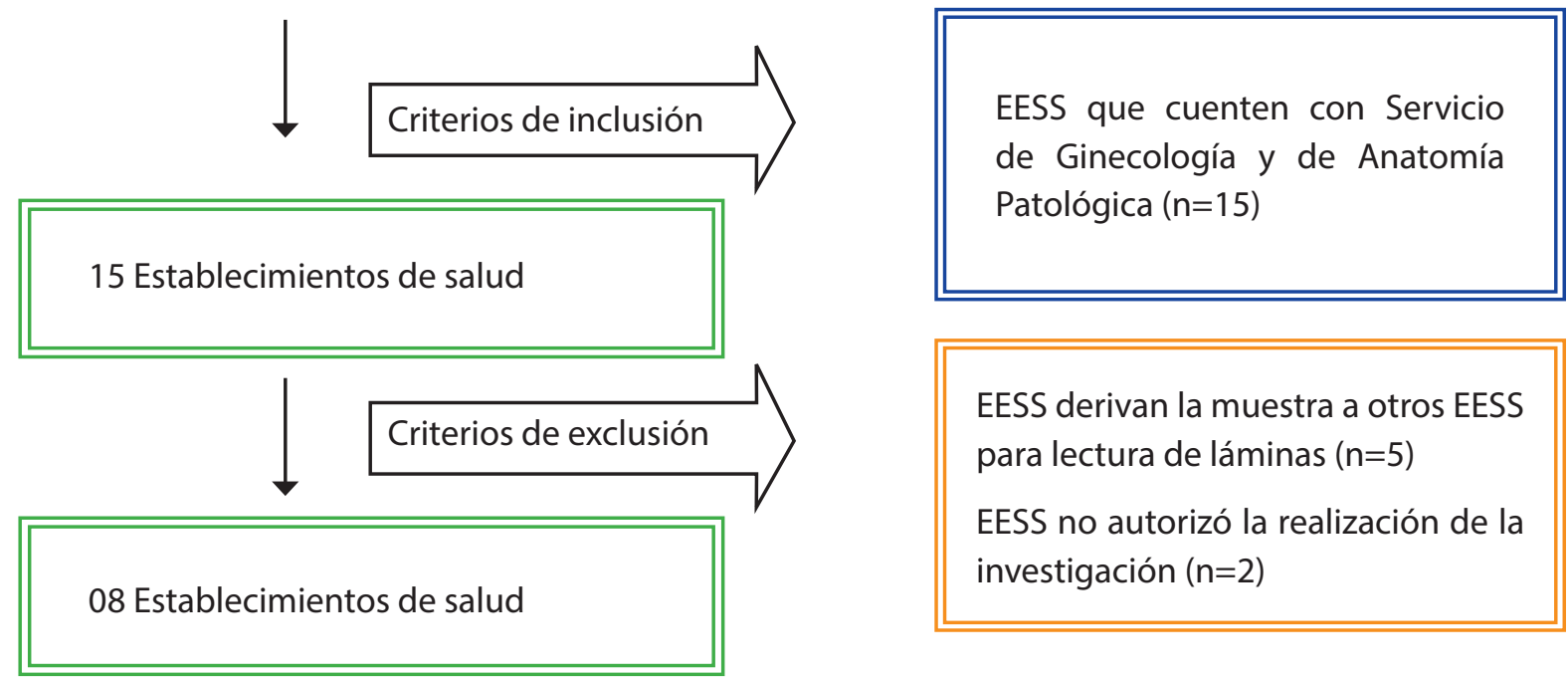

Figura 1. Árbol de Selección de Establecimientos de Salud participantes. 


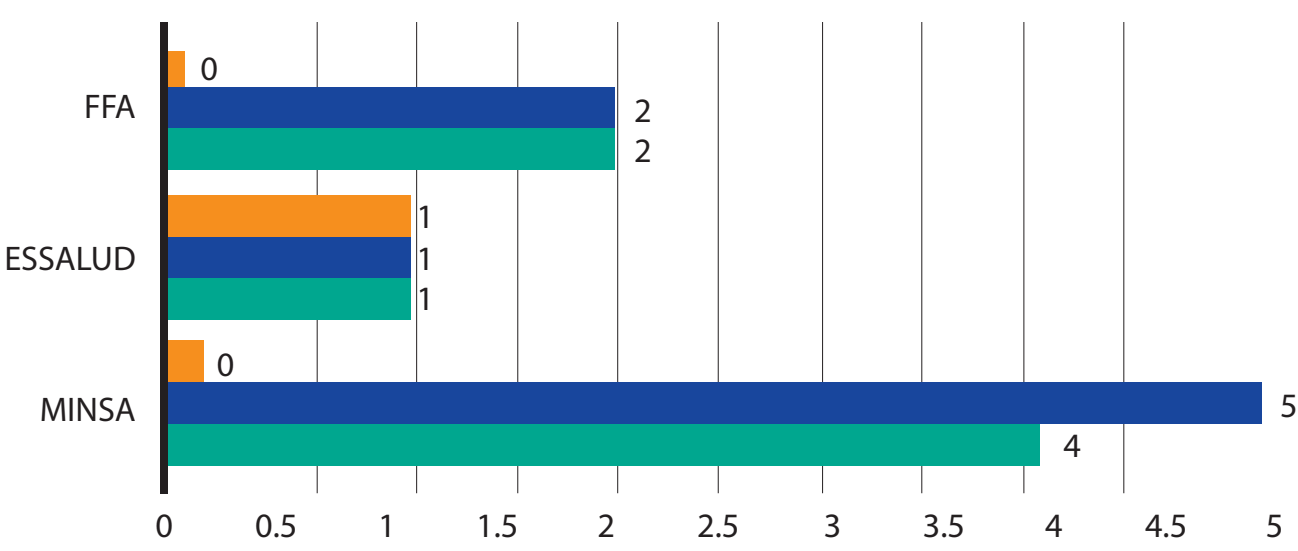

Gráfico 1. Profesional que extrae la muestra del tamizaje.

\section{DISCUSIÓN}

EI CCU es el cuarto cáncer más frecuente en el mundo², generando en países latinoamericanos, verbigracia Ecuador, un alto costo efectividad de $\$ 597$ por cada año de vida ajustado por discapacidad ${ }^{17}$. En el Perú, el costo estimado en el 2011 generado por el CCU fue de $\$ 15,5$ millones $^{18}$, siendo la primera causa de muerte en mujeres ${ }^{5}$ y por ello que a través del Ministerio de Salud, se han implementado diferentes estrategias y políticas que resaltan la importancia de la cobertura, la detección temprana y el tamizaje ${ }^{15}, y$ es respecto a éste último, que se estudió en $8 \mathrm{EESS}$ los hallazgos referentes al Tipo de Citología utilizada, al profesional que extrae, realiza la primera lectura y da el diagnóstico, el uso de la metodología de Bethesda en el Reporte Final, el uso de detección de ADN de VPH y el seguimiento de hallazgos ASCUS.

Se encontró que en todos los EESS $(n=8)$ el tamizaje de CCU se realiza mediante la Citología Convencional de Papanicolaou (CC) y en ningún caso mediante la Citología de Base Líquida (CBL). Esto concuerda con los hallazgos de reportes en países en vías de desarrollo en los cuales la CC es el método más utilizado ${ }^{6,19}$. Sin embargo, a pesar de sus ventajas e implementación, existen limitaciones en esta técnica que hay que tener en cuenta: empezando por problemas de cobertura a nivel nacional ${ }^{18}$, sensibilidad muy variable y falsos negativos de hasta $49 \%^{20,21}$ éstos últimos producto de errores en la toma de muestra, preparación o hasta de interpretación; por último es importante resaltar la dependencia que tiene este método con la calidad del Sistema de Salud del país en el que se implementa, al punto que estudios refieren que en sistemas poco organizados, con pocos recursos y poca oferta, el PAP no es de utilidad en la reducción de la incidencia del $\mathrm{CCU}^{22}$. Es por ello que, algunos estudios refieren que la tendencia a futuro es que los programas de tamizaje utilicen la $\mathrm{CBL}^{4}$ la cual posee una sensibilidad de hasta $80 \%$, reduciendo tanto en 70 a 90\% el índice de muestras no satisfactorias encontradas con la $\mathrm{CC}^{20}$, como otros problemas de la CC ya previamente descritos en la literatura ${ }^{23}$.

Además, esta técnica permite que la muestra sea sometida a análisis molecular de ADN de $\mathrm{VPH}^{24}$, permitiendo estudios de tipificación además, siendo considerada por algunos estudios como una alternativa viable y significativamente mejor en casi todos los aspectos en comparación a la $\mathrm{CC}^{25}$. Sin embargo, la principal desventaja de ésta radica en el aspecto económico e implementación ${ }^{20}$. Existen estudios que proponen que la CBL es una alternativa costo efectiva teniendo en cuenta el costo de una máquina para CBL en $\$ 50000$ y la comparación entre costos de insumo per cápita entre la CC y la CBL, de $\$ 2.5$ y $\$ 6$ respectivamente ${ }^{26}$. Esto se fundamenta en la reducción del número de falsos negativos, la cantidad de muestras insatisfactorias, disminución del tiempo para obtener y evaluar la muestra, la conservación de esta para estudios especializados posteriores ${ }^{27,28}$ y además, la reducción del tiempo para derivar a la paciente para la colposcopía y el tiempo de 
seguimiento y distanciamiento en hacer la siguiente citología cuando la citología y el VPH son negativos ${ }^{29}$.

El proceso del Tamizaje para CCU puede plantearse en 5 etapas ${ }^{11,30}$ : dos etapas a estudiar son la etapa pre-analítica que abarca el proceso de la extracción de muestras la cual es señalada por algunos estudios como la etapa en la que se producen la mayoría de errores $^{30}$, pudiendo perjudicar a las demás etapas del proceso $^{11}$, y la post analítica a la cual se le atribuyen el $9 \%$ de los errores ${ }^{30}$. En nuestro estudio, en la etapa preanalítica, la extracción de la muestra estuvo a cargo del Médico Cirujano Especialista en Ginecología y/o las Licenciadas en Obstetricia en 7 casos, existiendo en un EESS que éstas últimas realizan exclusivamente la extracción y en uno en el cual la muestra es extraída por el Médico Cirujano Especialista en Ginecología, las Licenciadas en Obstetricia y/o las licenciadas en enfermería. Independientemente del profesional que realice este proceso, es importante resaltar la experticia del profesional que la realiza y la necesidad de su evaluación continua por competencias a través de escalas validadas ${ }^{13}$, todo ello para contrarrestar el problema de los "tomadores ocasionales" definidos como personal que toma menos de 50 muestras al año ${ }^{13}$ y que por sus limitaciones en la técnica pueden contribuir a muestras inadecuadas que dan un alto índice de falsos negativos ${ }^{14}$.

En la fase analítica del tamizaje, se realiza la interpretación del resultado. En nuestro estudio se evidenció que en 07 EESS, la primera lectura de la muestra estuvo a cargo del Tecnólogo Médico, siendo 01 EESS en la cual está a cargo tanto de éste como del Anátomo Patólogo; y que además, en ningún caso se contó con un sistema automatizado para la primera lectura. La literatura menciona que la lectura puede ser realizada por un citólogo, citotecnólogo o un patólogo calificado ${ }^{11}$. Por otro lado, es importante resaltar que la Lectura Automatizada ${ }^{31}$ no está implementada aún en los EESS, y tener en cuenta los posibles beneficios que ésta traería como la reducción en 32\% del índice de falsos negativos ${ }^{32}$. Existen estudios que mencionan que, independientemente del tipo de citología, con el uso de Lectura Automatizada la mejora en el rendimiento y productividad del proceso en términos de duración de la lectura y número de muestras analizadas por hora o día, fue superior al manual ${ }^{33,34}$; sin embargo también existen estudios que indican que pese a la mayor productividad es menos coste efectiva en comparación a la lectura manual ${ }^{33}$.

Respecto del profesional que realiza el diagnóstico y además al uso del Sistema de Bethesda para el reporte final, en todos los EESS dicha función estuvo a cargo del Anátomo-Patólogo Especialista quien en todos los casos utilizaba el sistema mencionado; esto concuerda con recomendaciones internacionales en las que se indica aquello $0^{10,11,30}$ permitiendo manejar un sistema de lenguaje uniformizado, razonablemente reproducible y flexible a las condiciones ${ }^{16}$. Sin embargo, este beneficio debe ir acompañado de capacitación y actualización constante sobre el sistema dirigido tanto a los Especialistas de Anatomía Patológica y a los Ginecólogos, además de superar las opiniones de algunos autores respecto a lo poco práctico del sistema de Bethesda tanto para el uso del aprendizaje de citología ginecológica como para la correlación de los estadios y hallazgos citológicos ${ }^{30}$.

Respecto del uso de pruebas para detección del ADN del VPH en las muestras, se describe que en ningún EESS se realiza dicho procedimiento. Existen beneficios establecidos respecto a dicha técnica, los cuales parten desde una extracción sencilla hasta la inmediatez del inicio del tratamiento una vez establecido un resultado positivo ${ }^{35}$. Además, en algunos países y centros especializados se ha propuesta usar dicha detección dirigida a VPH de alto riesgo como un método de tamizaje primario ${ }^{16}$, logrando resultados de hasta un 60 a $70 \%$ de mayor protección contra el CCU, siendo más eficaz que el PAP y la prueba de ácido acético ${ }^{36}$, y alargando los intervalos de cribado hasta 5 años en mujeres con VPH negativo ${ }^{37}$. El Instituto Nacional de Salud a través de su Boletin del 2016, afirma que la detección molecular de VPH podría usarse ante hallazgos de tipo ASCUS, sin embargo su implementación en un Plan Nacional implicaría altos $\operatorname{costos}^{38}$.

Estudios en México señalan que las estrategias basadas en la detección de ADN de VPH, la tipificación del Virus, la CBL y la inmunotinción como procedimiento de triaje es una alternativa menos costosa en comparación con los costos per cápita del tratamiento de casos con NIC 2-3 y $\mathrm{CCU}^{24}$. Sin embargo, el costo unitario suele ser elevado y los requerimientos logísticos especializados suelen no ser accesibles ${ }^{35}$, existiendo estudios que la catalogan como altamente costo-efectiva con $\$ 3482$ $\mathrm{AVAD}^{39}$. Otro potencial beneficio es la identificación de los diferentes genotipos de VPH presentes en la población peruana, tal como lo planteado en algunos estudios con hallazgos inesperados para VPH-16 y otros VPH-AR ${ }^{40}$; lo cual permitiría la confección de un registro nacional y la evaluación del impacto de las estrategias de vacunación frente $\mathrm{VPH}^{41}$. Sobre ello, actualmente ya existen en países como México 
estudios como el denominado estudio FRIDA cuyo objetivo es evaluar el desempeño y costo-efectividad de diferentes alternativas de triage en mujeres VPH AR positivas $^{42}$.

Si bien el Sistema de Bethesda está recomendado por la OMS, existen alteraciones inespecíficas las muestras de células epiteliales denominadas ASCUS que representan un punto intermedio entre benignidad y malignidad, situación que no ha sido modificada en la última actualización de Bethesda del 2014; esto conforma un grupo de hallazgos importantes por su potencial malignidad ${ }^{43}$, alta cantidad de frecuencia de reportes en Citología Convencional' ${ }^{16}$ y cuyo seguimiento debería ser obligatorio. Frente a hallazgos ASCUS, las guías actualizadas recomiendan el Co-Test que incluye el PAP con pruebas de ADN de VPH de alto riesgo ${ }^{16}$. En este estudio se determinó que 7 EESS realizan el seguimiento de éstos hallazgos, lo cual indica que no en todos los EESS se han implementado estrategias que realcen la importancia de dicho proceso, a pesar de estar considerado dentro de la fase post post analítica del tamizaje para $\mathrm{CCU}^{11}$, ni considerando que existen reportes de tasas de falta de adherencia cercanas en algunos casos a más del $50 \%{ }^{44}$.

Como limitaciones, durante la elaboración del trabajo se identificó la falta de políticas institucionales en algunos EESS para el fomento del desarrollo de trabajos multicéntricos y la existencia de políticas que limitan el desarrollo de investigación por parte de investigadores externos a sus instituciones. Por parte del diseño y muestreo, las características del Universo de Estudio impidieron contar con información proveniente de otras Sedes Hospitalarias en Lima Metropolitana cuyos resultados hubieran contribuido al enriquecimiento del estudio, tal es el caso del Centro de Prevención Larco, el Hospital Cayetano Heredia y el Hospital Arzobispo Loayza por ejemplo. Además, algunos EESS realizan la toma de muestra y refieren el resultado a sus establecimientos de cabeza de Red, lo cual limita la descripción del primer componente de proceso del Tamizaje para CCU en dichos establecimientos, y al mismo tiempo, disminuye el número de EESS participantes del estudio.

\section{Recomendaciones}

Este estudio preliminar recomienda el desarrollo de estudios posteriores enfocados a la descripción de las fases del proceso de Tamizaje del CCU y en especial de la toma de muestra; estudios de costo-utilidad de implementación de la CBL y el Co-test, y también en la implementación de métodos automatizados de lectura de láminas. Además, este estudio puede ser replicado incluyendo un mayor número de EESS a nivel regional y nacional. Se recomienda el desarrollo de capacitaciones continuas y evaluaciones mediante escalas validadas a los diferentes profesionales que intervienen en el proceso de tamizaje, así como la capacitación conjunta entre Especialistas Anátomo Patólogos y Ginecólogos en torno a las actualizaciones del Sistema de Bethesda y su implementación.

\section{Agradecimientos}

Los autores agradecen la colaboración de las Sedes Hospitalarias y sus respectivos Jefes de Servicio que aceptaron participar en el estudio, al Instituto de Investigación en Ciencias Biomédicas - INICIB- de la Facultad de Medicina Humana de la Universidad Ricardo Palma, y a la Sociedad Científica Médico Estudiantil Peruana-SOCIMEP.

Contribuciones de autoría: Los autores participaron en la generación, recolección de información, redacción y aprobación final del artículo original.

Financiamiento: Autofinanciado.

Conflicto de interés: El autor Dr. Alejandro Yábar Berrocal es el Jefe de uno de los Servicios en donde se realizó el presente estudio.

Recibido: 19 de noviembre del 2018

Aprobado: 20 de diciembre del 2018

Correspondencia: Aleksandar Cvetkovic Vega

Dirección: Jr. Bronsino 307, San Borja, Lima-Perú

Celular: +51964982676

Correo:aleksandar.famurp@gmail.com 


\section{REFERENCIAS BIBLIOGRÁFICAS}

1. Cox JT. Epidemiology and natural history of HPV. J Fam Pract. 2006 Nov;Suppl:3-9.

2. Globocan. Cervical Cáncer: Estimated incidence, mortality and prevalence worldwide in 2012 [Internet]. 2012 [cited 2016 Oct 27]. Available from: http:// globocan.iarc.fr/old/FactSheets/cancers/cervix-new.asp

3. Galán-Rodas E, Vélez CD, Rodas JL. Tamizaje citológico para cáncer de cuello uterino: una problemática emergente. Rev Cuerpo Méd Hosp Nac Almanzor Aguinaga Asenjo. 2013;6(2):52-4.

4. Santos-Ortiz C, Manrique J, Amorín E, Sarria G, Salazar M, Limache A, et al. Acelerando la innovación en el control del cáncer en el Perú. Rev Peru Med Exp Salud Pública. 2016 Aug 4;33(3):535.

5. Bruni L, Barrionuevo-Rosas L, Alberto G, Serrano B, Mena M, Gómez D, et al Human Papillomavirus and Related Diseases in Peru. [Internet]. 2016 [cited 2016 Oct 27]. Available from: http://www.hpvcentre.net/statistics/reports/PER.pdf

6. Organización mundial de la salud. Perfiles oncológicos de los país: Perú [Internet] Perú; 2014 [cited 2016 Oct 27]. Available from: http://www.who.int/cancer/countryprofiles/per_es.pdf?ua $=1$

7. Instituto Nacional de Salud. Acta de Acuerdos y Compromisos: Taller de Identificación de prioridades de investigación en salud 2015-2021 DISAS LIMA SUR y LIMA ESTE. [Internet]. Perú; 2014. Available from: http://www.ins.gob.pe/ repositorioaps/0/2/jer/prior_reg/PRIORIDADES\%20lima\%20acta.pdf

8. Organización mundial de la salud. Papilomavirus humano ( $\mathrm{PVH}$ ) y cáncer cervicouterino [Internet]. 2015. Available from: http://www.who.int/mediacentre/ factsheets/fs380/es/

9. Zaharia M. El cáncer como problema de salud pública en el Perú. Rev Peru Med Exp Salud Pública. 2013;30(1):07-8.

10. Pelea CL. Nomenclatura de las lesiones cervicales (de Papanicolau a Bethesda 2001). Rev Esp Patol. 2003;36(1):5-10.

11. Arriaga VL, Gaspar DV. Control de calidad en Citología, Colposcopia y estudios Anatomopatológicos. Arch Méd Actual En [Internet]. 2012 [cited 2017 Feb 21] Available from: http://www.medigraphic.com/pdfs/archivostgi/tgi-2012/tgi126g.pdf

12. Williamson SLH, Hair T, Wadehra V. The effects of different sampling techniques on smear quality and the diagnosis of cytological abnormalities in cervical screening. Cytopathology. 1997;8(3):188-95.

13. Sandra Olimpia Gutiérrez-Enríquez, Darío Gaytán-Hernández, José Manuel Zamarripa-Leyva, Yolanda Terán-Figueroa. Desempeño del personal de salud en la toma de las citologías cervicales: conocimientos teóricos y ejecución práctica [Internet]. Revista de Ginecología y Obstetricia de México; 2014. Available from: http://www.medigraphic.com/pdfs/ginobsmex/gom-2014/gom141c.pdf

14. Georgina O, Irma H. Análisis del descenso de la mortalidad por cáncer cervicouterino en el IMSS, 1991-2005. Rev Med Inst Mex Seguro Soc. 2006;44(Supl 1):S129-134.

15. Sarria-Bardales G, Limache-García A. Control del cáncer en el Perú: un abordaje integral para un problema de salud pública. Rev Peru Med Exp Salud Pública. 2013;30(1):93-8.

16. Nayar R, Wilbur DC. The Pap Test and Bethesda 2014. Acta Cytol. 2015 May 19;59(2):121-32.

17. Organización mundial de la salud, Organización panamericana de la salud. Directrices de la OPS/OMS sobre tamizaje y tratamiento de lesiones precancerosas para prevención del CCU.pdf. 2013.

18. Alfonso Gutiérrez-Aguado. Costo-utilidad de la vacuna contra el virus de papiloma humano en mujeres peruanas [Internet]. Revista Peruana de Medicina Experimental y Salud Pública; 2011 [cited 2016 Oct 27]. Available from: http://www. scielosp.org/scielo.php?script=sci_arttext\&pid=S1726-46342011000300003

19. Sharma J, Toi P, Siddaraju N, Sundareshan M, Habeebullah S. A comparative analysis of conventional and SurePath liquid-based cervicovaginal cytology: A study of 140 cases. J Cytol. 2016;33(2):80.

20. Berek JS, Novak E. Berek \& Novak's gynecology. Philadelphia: Wolters Kluwe Health/Lippincott Williams \& Wilkins; 2012

21. Santiago Alonso, Gema Vaquero, Rocío Montejo, Manuela López, Pilar Miranda Monserrat López, et al. Comparación en el rendimiento diagnóstico de lesiones cervicales entre la citología convencional de Papanicolaou y la citología en base líquida, en una misma población de referencia. In Granada, España; 2006.

22. Goldie SJ, Gaffikin L, Goldhaber-Fiebert JD, Gordillo-Tobar A, Levin C, Mahé C, et al. Cost-effectiveness of cervical-cancer screening in five developing countries. N Engl J Med. 2005;353(20):2158-68.

23. Ricci P, Perucca E, Koljanin J, Baeriswyl E. Citología de base líquida: revisión de la historia y los estudios al respecto. Rev Chil Obstet Ginecol. 2004;69(3):256-62.

24. Beal CM, Salmerón J, Flores YN, Torres L, Granados-García V, Dugan E, et al. Cost analysis of different cervical cancer screening strategies in Mexico. Salud Pública México. 2014;56(5):429-501.

25. Bak M, Séberné Éll M, Bóka M, Veleczki Z, Nyári T, Pete I, et al. Liquid-based cervical cytology ThinPrep screening in Hungary. Orv Hetil. 2014 May;155(18):708-14.

26. Cox JT. Liquid-based cytology: evaluation of effectiveness, cost-effectiveness, and application to present practice. J Natl Compr Canc Netw. 2004;2(6):597-611.

27. Guo J, Cremer M, Maza M, Alfaro K, Felix JC. Evaluation of a Low-Cost LiquidBased Pap Test in Rural El Salvador: A Split-Sample Study. J Low Genit Tract Dis. 2014 Apr;18(2):151-5.

28. Alameda F, Fusté $P$, Albert $S$, Romero E, Gimferrer E, Soler I, et al. Citología en medio líquido (Thin Prep Pap Test). Un año de experiencia. Prog Obstet Ginecol. 2007 Apr;50(4):197-202.

29. Olry de Labry Lima A, Epstein D, García Mochón L, Ruiz Aragón J, Espín Balbino J. Análisis de coste-efectividad de la prueba de citología cervicovaginal. Prog Obstet Ginecol. 2012 Aug;55(7):304-11.

30. Moya-Salazar J, Rojas-Zumaran V, Torres-Martínez R, Rosas-Vargas L. Calidad de los extendidos cervicouterinos dentro de la coloración de Papanicolaou para el cribado de cáncer cervical en Lima, Perú. Rev Esp Patol. 2016 Jan;49(1):7-18.

31. Rodríguez-Vázquez S. Support to the Diagnosis of the Pap Test, Using Computer Algorithms of Digital Image Processing. In: Sidorov G, Herrera-Alcántara O, editors. Advances in Computational Intelligence [Internet]. Cham: Springer International Publishing; 2017 [cited 2017 Sep 16]. p. 425-36. Available from: http://link.springer. com/10.1007/978-3-319-62434-1_35

32. Bansal B, Gupta P, Gupta N, Rajwanshi A, Suri V. Detecting uterine glandular lesions: Role of cervical cytology. CytoJournal. 2016;13(1):3.

33. De Tecnologías Sanitarias I de E. Métodos automatizados de lectura de citología cervical uterina. [cited 2016 Oct 29]; Available from: http://www.sergas.es/gal/ publicaciones/docs/avalia-t/pdf-2236-ga.pdf

34. Eurocytology. Guías para la organización de laboratorio, procesamiento y screening [Internet]. [cited 2017 Feb 21]. Available from: http://www.eurocytology. eu/es/course/486

35. World Health Organization, World Health Organization, Reproductive Health and Research. Comprehensive cervical cancer control: a guide to essential practice. [Internet]. 2014 [cited 2016 Oct 27]. Available from: http://www.ncbi.nlm.nih.gov/ books/NBK269619/

36. Vargas-Hernández VM, Vargas-Aguilar VM, Tovar-Rodríguez JM. Detección primaria del cáncer cervicouterino. Cir Cir. 2015 Sep;83(5):448-53.

37. De Sanjosé S. Cambios en el cribado del cáncer de cuello uterino. Aten Primaria. 2016 Nov;48(9):563-4.

38. Hijar G. Detección molecular y genotipificación de virus del papiloma humano como tamizaje de cáncer de cuelo uterino: posibilidades en el contexto peruano. Bol-Inst Nac Salud. 2016;22(1-3):22-8

39. Henríquez-Trujillo R, Narváez-Moscoso F. Estimación de la carga de enfermedad por cáncer de cuello uterino en Ecuador. Rev Médica. 2016;27(1):53-5.

40. Iwasaki Ricardo, Arias-Stella Javier Jr., Arias-Stella Javier. Prevalencia del virus de Papiloma Humano de alto riesgo en el Perú. Diagnóstico. 2014;53(1):5-8.

41. Marzo-Castillejo Merce B-BB, Vela-Vallespín Carmen, Nuin-Villanueva Marian, Bartolomé-Moreno Cruz, Melus-Palazón Elena, Vilarrubi-Estrella Merce. Recomendaciones de prevención de cáncer. Aten Primaria. 2016;48(1):39-59.

42. Group FS, others. Triage strategies in cervical cancer detection in Mexico: methods of the FRIDA Study. Salud Pública México. 2016;58(2):197-210.

43. Solom,D., Schiffman,M, Tarone, R. For the ASCUS/LSIL Triage Study for Cervical Cancer (ALTS) Group. Comparison of three management strategies for patients with atypical squamous cells of undetermined significance. Baseline results from a randomized trial. J Natl Cancer Inst. 2001;92(12):293-9.

44. Barrios L, Retamoso E, Alvis LR. Adherencia al seguimiento y evolución de la lesión en mujeres con Neoplasia intra epitelial cervical escamosa grado 1. Rev Colomb Cancerol. 2017 Jan;21(1):19-25. 